\title{
Patient-reported outcome measures in hemodialysis patients: results of the first multicenter cross-sectional ePROMs study in France
}

Abdallah Guerraoui ${ }^{1 *}$, Mathilde Prezelin-Reydit ${ }^{2}$, Anne Kolko $^{3}$, Marie Lino-Daniel ${ }^{1}$, Charlotte Dumas de Roque ${ }^{2}$, Pablo Urena ${ }^{3}$, Philippe Chauveau ${ }^{2}$, Catherine Lasseur ${ }^{2}$, Julie Haesebaert ${ }^{4}$ and Agnes Caillette-Beaudoin ${ }^{1}$

\begin{abstract}
Background: Kidney failure with replacement therapy and hemodialysis are associated with a decrease in quality of life (QOL). Self-reported QOL symptoms are not always prioritized by the medical team, potentially leading to conflicting priorities with patients. Electronic patient-reported outcome measures (ePROMs) allow physicians to better identify these symptoms. The objective was to describe the prevalence of symptoms self-reported by hemodialysis (HD) patients.
\end{abstract}

Methods: A multicenter cross-sectional study was conducted in three HD centers. Patients were included if they were 18 years old or over treated with HD for at least 3 months in a center. Data were collected by the patient via a self-administered ePROMs questionnaire. Data included patient characteristics, post-dialysis fatigue and intensity, recovery time after a session, perceived stress, impaired sleep the day before the dialysis session, current state of health and the change from the past year. A multivariate analysis was conducted to identify relations between symptoms.

Results: In total, we included 173 patients with a mean age of 66.2 years, a mean \pm SD hemodialysis duration of $48.9 \pm 58.02$ months. The prevalence of fatigue was $72 \% .66 \%$ had a high level of stress (level B or C). Recovery time was more than $6 \mathrm{~h}$ after a HD session for $25 \%$ of patients and $78 \%$ declared they had a better or unchanged health status than the previous year. Sleep disturbance was associated with cardiovascular comorbidities $(\mathrm{OR} 5.08$ [95\% Cl, 1.56 to 16.59$], p=0.007)$.

Conclusions: Fatigue and stress were the main symptoms reported by HD patients. The patient's care teams should better consider these symptoms.

Keywords: Chronic kidney disease, Fatigue, Hemodialysis, Quality of life, Patient reported outcome, PROMs

*Correspondence: abdallah.guerraoui@calydial.org

${ }^{1}$ Calydial Dialysis Department, Calydial, CH Vienne Lucien Hussel, Lucien Hussel Hospital, Vienne, France

Full list of author information is available at the end of the article

\section{Background}

Patients with chronic kidney disease (CKD) treated with hemodialysis (HD) require long-term care with a potentially negative impact on their quality of life (QoL) [1]. Patients treated with hemodialysis have a high symptomatic burden additionally impacting their QoL, which include pain, fatigue and stress [2]. 
These symptoms are, however, not always considered as a priority focus by the medical team caring for such patients and thus are not routinely collected despite their relevance to patients $[3,4]$. The lack of physician focus on QoL may lead to conflicting medical and treatment priorities between a patient and their care team and potentially lead to undiagnosed and untreated symptoms. This is especially apparent for patients on maintenance dialysis [5-7].

The collection of electronic patient-reported outcome measures (ePROMs) is an innovative method to better take consider these symptoms [8]. Moreover, ePROMs allow patients to report their symptoms on a regular basis and allow the medical team to adapt treatment plans accordingly [8]. However, the use of ePROMs for CKD patients treated with HD in routine care remains limited $[9,10]$. The objective of the study was to describe the prevalence of symptoms reported by patients treated with hemodialysis using ePROMs. The secondary objective was to explore predictive factors of the presence of the patient reported symptoms.

\section{Methods}

\section{Study design}

A multicenter cross-sectional observational study was conducted in three hemodialysis centers in France (Vienne, Bordeaux and Paris) between January and March 2020. Vienne regularly followed 90 patients, Bordeaux 128 and Paris 149. Patients were included if they were aged at least 18 years old and treated with hemodialysis for at least 3 months in one of the participating centers. Patients were excluded if they refused to participate in the study or could not read or understand French well enough to complete the questionnaires.

\section{Choice of symptoms}

We chose to report the symptoms which were the most frequently reported in a preliminary qualitative study. This qualitative study was conducted to identify relevant symptoms reported by patients and affecting quality of life between dialysis sessions. A total of 20 patients were interviewed by a nephrologist trained to conduct semi-structured interviews. Patients were encouraged to provide examples and expand their answers to collect further details. Interviews were then transcribed and coded with thematic analysis to identify theme and subthemes from the data. Interviews were ceased when no new codes were identified and data saturation was reached [11]. We explored inter-dialysis symptoms and selected the three most frequent ones that were fatigue, stress-related symptoms and sleep disorders (Table 1). The interview guide, patient details and verbatim are provided in Additional file 1.

\section{Data collection}

ePROMs data were collected at one time with a selfadministered by the patient ePROMs through an
Table 1 Themes and subthemes identified during the preliminary qualitative phase with for patients with chronic kidney disease (CKD) treated with hemodialysis in the ePROMs study

\begin{tabular}{ll}
\hline Themes & Subthemes \\
\hline Fatigue & Feeling tired \\
& Lack of energy \\
Mental symptoms & Feeling anxious \\
& Feeling irritable \\
& Feeling sad \\
& Feeling nervous \\
& Concern \\
Sleep disorders & Difficulty falling asleep \\
& Difficulty staying asleep \\
Other symptoms & Decreased appetite \\
& Decreased sexual desire \\
& Dry mouth \\
& Cramps \\
& Itching \\
\hline
\end{tabular}

electronic tablet during a HD session or consultation by the patient or with the help of a caregiver if necessary. The collection of data through a tablet was chosen due to the ease of use and simple interface for the patients. The validation of the questionnaire required the questionnaire to be completed therefore there could not be any missing data.

Data collected included the presence of post-dialysis fatigue with a binary question (yes/no), its perceived intensity with a visual analog scale (VAS) ranged from 0 to 10 ( 0 was no perceived intensity and 10 was the most possible perceived intensity) [12], and the recovery time of this symptom after a session as a Likert scale question [13] (Table 2). Perceived stress (PSS 10) was evaluated using a scale adapted from Cohen and Williamson $[14,15]$ (Table 2). Sleep quality the day before the dialysis session was evaluated in the form of a Likert scale format, the current state of health of the patient using a VAS $[16,17]$, and the one-year change using a Likert scale were also collected. Patient characteristics were described from the patient's medical records (demographics, dialysis situation, BMI, comorbidities, hemoglobin). Ethnicity was not collected in compliance with national regulations.

\section{Statistical analysis}

We included all patients with eligibility criteria during the study period, our aim was to include all patients during a 3 month period to be representative of patients. Continuous variables were described using means \pm 
Table 2 Symptom scales chosen for patients with chronic kidney disease treated with hemodialysis in the ePROMs study

\begin{tabular}{|c|c|c|}
\hline Symptoms & Indicator & Scale \\
\hline \multirow[t]{3}{*}{ Fatigue } & Prevalence & Binary question: Yes/No \\
\hline & Intensity & Visual analog scale from 0 (not tired) to 10 (very tired) \\
\hline & Recovery time & Likert scale (less than $2 \mathrm{~h}, 2$ to $6 \mathrm{~h}, 7$ to $12 \mathrm{~h}$, more than $12 \mathrm{~h}$ ) \\
\hline \multirow[t]{2}{*}{ Stress } & Intensity & Visual analog scale from 0 (not stressed) to 10 (very stressed) \\
\hline & Severity & $\begin{array}{l}\text { Perceived Stress Scale adapted from Cohen and Williamson } \\
\text { A }(<21) \text { : knows how to manage stress and adapt to find solutions } \\
\text { B ( } 21 \text { to } 26) \text { : knows to manage stress most of the time but it is not possible to manage stress in } \\
\text { some situations. It is possible to learn stress management techniques } \\
C(\geq 27) \text { : does not know how to manage stress and perception of continuous threat which can } \\
\text { negatively impact life and disease course. }\end{array}$ \\
\hline Sleep disturbance & $\begin{array}{l}\text { Sleep quality the night before } \\
\text { dialysis compared to other } \\
\text { nights }\end{array}$ & $\begin{array}{l}\text { Likert scale (My sleep is better, my sleep is more or less good, my sleep is unchanged, my sleep } \\
\text { is altered) }\end{array}$ \\
\hline \multirow[t]{2}{*}{ Overall health status } & Current status & Visual analog scale from 0 (very poor health) to 10 (very good health) \\
\hline & Comparison to 1 year before & Likert scale (poorer status, unchanged, more or less improved, improved) \\
\hline
\end{tabular}

standard deviations (SD) or medians and interquartile ranges, according to the normality of their distribution. Numbers and percentages were used for qualitative variables. We first described the prevalence of each ePROM with an estimated 95\% confidence interval [95\% CI]. We compared patient characteristics and ePROMS between the three participating centers. Association between ePROMs (recovery time of fatigue ( $\leq 6-\mathrm{h}$ versus $>6-\mathrm{h}$ ), perceived stress scale (PSS) stress level (A versus BC), sleep quality (disrupted if answer was "altered" versus not disrupted) and patients' characteristics and clinical variables was explored using chi square comparisons or Student's $\mathrm{t}$ and Wilcoxon tests according to the nature and distribution of the variables.

Multivariate logistic regression models were conducted to identify if patient characteristics or clinical variables were associated with recovery time of fatigue (Model 1), perceived stress using PSS (Model 2) and sleep quality (Model 3). Variables included in the model were chosen based on available literature, expert discussion and results of the bivariate analysis. A multivariate regression model was built for each e-PROMS, with the ePROMs as dependent variable, and clinical characteristics (age, gender, dialysis duration and cardiovascular history), undernutrition, hemoglobin, duration of hemodialysis session and number of sessions per-week, and the two other e-PROMs as independent variables. Undernutrition was defined by at least two of three following criteria: Serum Albumin <35 g/l, Serum Prealbumin <300 mg/l, nPCR $<1.2 \mathrm{~g} / \mathrm{Kg} / \mathrm{d}$. Cardiovascular history was defined by at least one of the following: diabetes, coronary artery disease, heart failure and stroke. A bilateral threshold of 5\% was considered to define the statistical significance. The analysis was performed with SAS 9.1 software.

\section{Ethical considerations}

The study was declared to the data protection authority in France, known as the Commission Nationale de l'Informatique et des Libertés (CNIL), and was approved by the CPP Ile-de-France VII on 26 December 2019 in accordance with French regulations.

\section{Results}

In total, 173 patients were included during the study period. The mean age of patients was $66.22 \pm 14.4$ years and majority of patients were males (68\%). Patients were treated with hemodialysis for a mean total duration of $48.9 \pm 58.02$ months (median: 31 months) and were mainly treated in self-dialysis unit (67\%). The mean length of dialysis per week was $11.46 \pm 1.41 \mathrm{~h}$ corresponding to $3.09 \pm 0.56$ sessions per week (Table 3 ).

In terms of comorbidities, $73 \%$ of patients had at least one comorbidity with coronary artery disease (34\%), congestive heart failure (34\%), diabetes (28\%), cancer (28\%) and undernutrition (16\%). Expected differences between centers were observed (shown in Table 3). Regarding laboratory test results, $70 \%$ of patients had hemoglobin $(\mathrm{Hb})$ between 10 and $12 \mathrm{~g} / \mathrm{dL}$, and $8 \%$ had $\mathrm{Hb}$ inferior to $10 \mathrm{~g} /$ $\mathrm{dL}$. Moreover, $13 \%$ of patients had their albumin inferior or equal to $35 \mathrm{~g} / \mathrm{dL}$, and $29 \%$ has single-pool $\mathrm{Kt} / \mathrm{V}$ inferior or equal to 1.2 .

The prevalence of fatigue was $72 \%$ [95\% CI, 64.7 to $78.7 \%$ ] with a mean severity score of $5.84 \pm 2.12$ on a zero to ten scale. Recovery time was more than $6 \mathrm{~h}$ for $25 \%$ [ $95 \%$ CI, 18.6 to $32 \%$ ] of patients. 39\% [95\% CI, 32 to $47 \%$ ] of patients have a stress level $\mathrm{C}$ (which meant that they did not know how to manage stress and perception of continuous threat which can negatively impact life and disease course). $27 \%$ [95\% CI, 20 to 35\%] of patients have 
Table 3 Patient characteristics with chronic kidney disease (CKD) treated with hemodialysis in the ePROMs study

\begin{tabular}{|c|c|c|c|c|c|}
\hline & Total & Center 1 & Center 2 & Center 3 & $p$-value \\
\hline Patients n (\%) & $173(100)$ & $72(41.6)$ & $44(25.4)$ & $57(33)$ & \\
\hline Age mean $( \pm S D)$ & $66.2 \pm 14.4$ & 71.8 & 58.7 & 64.5 & $<0.001$ \\
\hline Gender (Male) n(\%) & $117(68)$ & $50(69)$ & $31(70)$ & $36(63)$ & 0.674 \\
\hline \multicolumn{6}{|l|}{ Dialysis } \\
\hline Duration in months mean (median) & $48.9(31)$ & $35(26.5)$ & $62.9(30)$ & $56.9(38)$ & 0.107 \\
\hline Self dialysis unit (\%) & 67 & 44 & 61 & 100 & $<0.001$ \\
\hline Duration of session & $11.5 \pm 1.4$ & $11.1 \pm 1.5$ & 12 & $11.6 \pm 0.6$ & 0.001 \\
\hline Number of sessions per week & $3.1 \pm 0.6$ & $3 \pm 0.5$ & $3.2 \pm 0.6$ & 3.20 .6 & 0.011 \\
\hline \multicolumn{6}{|l|}{ BMI n (\%) } \\
\hline$<18$ & $10(6)$ & $4(5,6)$ & $4(10)$ & $2(3,6)$ & 0.01 \\
\hline $18-25$ & $73(44)$ & $22(30,6)$ & $22(58)$ & $29(52,8)$ & \\
\hline$>25$ & $82(50)$ & $46(64)$ & $12(32)$ & $24(43,6)$ & \\
\hline \multicolumn{6}{|l|}{ Comorbidities \% } \\
\hline Undernutrition & 16 & 21 & 22 & 5 & 0.029 \\
\hline Diabetes & 28 & 43 & 16 & 16 & $<0.001$ \\
\hline Cerebrovascular disease & 6 & 10 & 6 & 2 & 0.162 \\
\hline Coronary artery disease & 34 & 48 & 18 & 28 & 0.004 \\
\hline Peripheral artery disease & 22 & 32 & 5 & 19 & 0.005 \\
\hline Congestive heart failure & 34 & 45 & 13 & 35 & $<0.001$ \\
\hline Cancer & 28 & 46 & 24 & 11 & $<0.001$ \\
\hline \multicolumn{6}{|l|}{ Hemoglobin (g/dL) } \\
\hline Mean $( \pm S D)$ & $11.26 \pm 1.1$ & $10.99 \pm 1.33$ & $11.60 \pm 0.75$ & $11.41 \pm 0.95$ & $<0.001$ \\
\hline$<10$ & $14(8)$ & $13(18)$ & $0(0)$ & $1(2)$ & \\
\hline 10 to 12 & $117(70)$ & $48(67)$ & $25(66)$ & $44(77)$ & \\
\hline$>12$ & $36(22)$ & $11(15)$ & $13(34)$ & $12(21)$ & \\
\hline \multicolumn{6}{|l|}{ Albumin (g/dL) } \\
\hline Mean $( \pm S D)$ & $39.6 \pm 5.9$ & $39.2 \pm 3.8$ & $39.7 \pm 3.9$ & $40.2 \pm 2.9$ & 0.004 \\
\hline$\leq 35$ & $21(13)$ & $14(19)$ & $6(16)$ & $1(2)$ & 0.009 \\
\hline$>35$ & $146(87)$ & $58(81)$ & $32(84)$ & $56(98)$ & \\
\hline \multicolumn{6}{|l|}{ Single-pool Kt/N } \\
\hline Mean $( \pm S D)$ & $1.44 \pm 0.34$ & $1.59 \pm 0.37$ & $1.09 \pm 0.21$ & $1.22 \pm 0.25$ & $<0.001$ \\
\hline$\leq 1.2$ & $48(29)$ & $12(17)$ & $3(8)$ & $33(58)$ & $<0.001$ \\
\hline$>1.2$ & $118(71)$ & $60(83)$ & $34(92)$ & $24(42)$ & \\
\hline
\end{tabular}

a stress level B (they know to manage stress most of the time but it is not possible to manage stress in some situations). The average intensity score was 3.3 on a zero to ten scale.

Sleep quality was disrupted for $15 \%$ [95\% CI, 9.6 to 20.6\%] of patients. The self-perceived health status of patients was $6.2 \pm 2.12$ (on a zero to ten scale) and $78 \%$ [95\% CI, 70.5 to $83.5 \%$ ] of patients stated that they had not a worsened health status than the year before (Table 4). No statistical differences were observed between centers for the three e-PROMS.

Results of the multivariate analysis are reported in Appendix 1. Fatigue recovery superior to $6 \mathrm{~h}$ was associated with the decreasing duration of HD sessions (OR
0.15; [95\% CI, 0.04 to 0.58 ] $p=0.006$ ) and with a higher stress level (OR 2.68; [95\% CI 1.04 to 6.88], $p=0.041$ ). Higher stress level was associated with female gender (OR 2.27; [95\% CI 1.001 to 5.14], $p=0.05$ ) and fatigue recovery superior to $6 \mathrm{~h}$ (OR 2.7; [ $95 \% \mathrm{CI}, 1.05$ to 6.92], $\mathrm{p}=0.04)$. Sleep disturbance was associated with cardiovascular comorbidities (OR 5.08; [95\% CI, 1.56 to 16.59], $p=0.007)$.

\section{Discussion}

In this study, we identified a high prevalence of selfreported fatigue at $72 \%$ and important stress at 39\% for CKD patients treated with hemodialysis. To our 
Table 4 Patients with chronic kidney disease (CKD) treated with hemodialysis reported symptoms in the ePROMs study

\begin{tabular}{lllll}
\hline & Total & Center 1 & Center 2 & Center 3 \\
\hline Fatigue $\mathrm{n}$ (\%) & $124(72)$ & $52(72)$ & $30(70)$ & $42(74)$ \\
Fatigue intensity & $5.8 \pm 2.1$ & $6 \pm 2.4$ & $5.8 \pm 1.9$ & $5.7 \pm 1.9$ \\
Recovery delay (\%) & & & & \\
$<2 \mathrm{~h}$ & 42 & 38 & 50 & 42 \\
2 to 6h & 33 & 32 & 34 & 33 \\
7 to 12h & 13 & 15 & 7 & 16 \\
$>$ 12h & 12 & 15 & 9 & 9 \\
Health status & $6.2 \pm 2.1$ & $6 \pm 2.5$ & $6.2 \pm 1.8$ & $6.5 \pm 1.9$ \\
Health status com- & & & & \\
pared to 1 year before & & & & \\
Improved & 40 & 40 & 39 & 41 \\
More or less improved & 9 & 11 & 9 & 5 \\
Non changed & 29 & 22 & 33 & 35 \\
Altered & 22 & 27 & 19 & 19 \\
Stress intensity & $3.7 \pm 3$ & $3.5 \pm 3.2$ & $3.3 \pm 3$ & $3.8 \pm 2.9$ \\
Stress severity (\%) & & & & \\
A & 34 & 29 & 36 & 37 \\
B & 27 & 29 & 25 & 26.32 \\
C & 39 & 42 & 39 & 37 \\
Sleep quality (\%) & & & & \\
Improved & 6 & 6 & 7 & 7 \\
More or less good & 20 & 18 & 18.5 & 23 \\
Non changed & 59 & 61 & 63 & 54 \\
Altered & 15 & 15 & 11.5 & 16 \\
\hline
\end{tabular}

knowledge, this was the first study in France to assess ePROM questionnaires filled directly with a tablet by patients in a dialysis unit.

Fatigue was the most prevalent symptom identified in our study, in comparison to the other symptoms assessed. The prevalence of $72 \%$ was consistent with the range of previous published literature, which presented results from 60 to $97 \%$. These results were additionally similar to the weighted mean prevalence of $71 \%$ estimated in a systematic review [18-20]. These results did not differ between centers even though patient characteristics and comorbidities differed.

Items collected from a patient were with a simple binary question and VAS instead of a dedicated measure and thus may not have reflected the specificity of fatigue from patients under hemodialysis. Such specific questionnaire was not available at the time of protocol definition and therefore a generic questionnaire was used. In future studies, items may be collected via the recently published measure SONG-HD questionnaire specifically designed for patients treated with hemodialysis [21]. This innovative tool designed through an international study included several components of fatigue including tiredness, lack of energy and inability to participate in social situations [22], however, did not distinguish between interdialytic fatigue and post-dialysis fatigue [23].

In our study, the post-dialysis fatigue through the aftersession delay recovery time in hours was chosen to be assessed as expressed by the patient. The recovery time inferior to $6 \mathrm{~h}$ found in the study for $75 \%$ of patients were similar compared to an international study where $73 \%$ of patients declared the same timing, as well as a recovery time of more than $12 \mathrm{~h}$ declared by $12 \%$ of patients from our study compared to $10 \%$ in the international DOPPS study [24]. Increased fatigue and higher levels of perceived stress were associated in the multivariate analysis indicating the potential interrelation between these two symptoms.

The stress assessment through the perceived stress score seemed to be more informative than the VAS to assess its intensity as it allowed to better discriminate patient groups with different stress levels. The two scales are different as the VAS describe the stress at the time of the questionnaire while the PSS includes the stress felt in the past week. Additionally, the VAS may reflect the stress level more at the time of the questionnaire while the PSS findings may reflect the stress tendency over the past weeks more and thus may be a better estimation of the patient stress level at home. The high proportion of patients with a stress level $\mathrm{C}$ may indicate the need to provide patients with interventions to better manage their stress and improve clinical outcomes.

Since stress and fatigue were also related in the multivariate analysis, having a positive impact on one of the symptoms may also have a positive impact on the other. Further research to better understand the interrelations between fatigue and stress and characterize the profiles of patients that are more prone to experience high level of these symptoms may therefore be necessary. Furthermore, women were more prone to higher level of stress in our study. Therefore, gender could also affect dialysis outcomes and additional research is necessary to better tailor adapted educational interventions.

Results on sleep quality were different to those reported in the literature. While only $15 \%$ of patients with reported altered sleep were found, sleep disturbance was reported at weighted mean prevalence of $44 \%$ with a range of 20 to $83 \%[12,25,26]$ in various countries. The observed difference may be due to the questionnaire used to assess the sleep quality in our study. The questionnaire in our study focused on the sleep quality the night before the dialysis session in comparison to the previous nights, to assess the potential impact of pre-dialysis anxiety on sleep quality. Additionally, these results may be in favor of a limited impact of the dialysis on sleep quality. This 
difference with literature could be explained because the primary indicator has been the disturbance in overall sleep quality for patients treated by hemodialysis in comparison to their situation before the initiation of a dialysis [27]. Moreover, the relation identified in our findings between sleep disorders and cardiovascular comorbidities matches with the literature on interrelations between sleep and cardiovascular diseases [28, 29].

The main strengths of this study relied on the crosssectional and multicenter design from various HD center settings and the use of simple questionnaires to collect data from patients through a tablet directly at patient side. The collection of data from patients during a consultation or HD session through a tablet device also allowed to not have missing data that could have weaken the interpretation of the results. On the other hand, the answers provided by patients may as well have been influenced by their location in the dialysis unit.

Despite significant differences in patient characteristics from the three centers including age, comorbidities or type of dialysis, no differences were found on the prevalence of the various PROMs, in favor of internally coherent results. The study population was not matching with the population profile of the French Renal Epidemiology and Information Network (REIN) [30-32] and consequently, in terms of comorbidities, coronary artery disease, congestive heart failure and cancer comorbidities the prevalence were higher in our study population. Contrarily, prevalence of diabetes and cerebrovascular disease were lower compared to REIN. This difference may lead to a selection bias and therefore our results may not be applicable to all hemodialysis patients in France. The 3 centers in our study have different characteristics, and no statistical differences were observed between centres for the three e-PROMS.

The main limitations of this study included the observational design, limited number of patients included, and the absence of linkage of PROMs with clinical outcomes such as cardiovascular events, hospitalizations or mortality. However, the objective was to first describe the symptoms of patients and not to explain these symptoms with their clinical situation or outcomes. It is also worth noting that this study did not assess whether better knowledge of patient reported symptoms could lead to better management of those symptoms by the care team, as it was noted in a recent study in Netherlands [33]. In terms of feasibility, time for completion and patient acceptability of the data collection process were also not collected.

Nonetheless, meta-analysis of oncology trials identified baseline fatigue as an independent prognostic factor for overall survival above performance status and quality of life in oncology patients, recommending collecting this information in routine oncology care for patient stratification [34]. Due to the clinical impact of fatigue on daily QoL of patients undergoing hemodialysis, it may be however relevant to consider the presence of reported fatigue in such patients to be a clinically relevant item to consider as itself, despite the need for further research in this area [35]. Additionally, recent studies identified an association between fatigue and all-cause mortality in those patients as well as between frailty and worse health related QoL [24, 36, 37].

To improve daily routine care of CKD patients treated with $\mathrm{HD}$, the collection and integration of ePROMs into the care plan could be promoted in a standardized approach. Such efforts are currently being conducted in various countries or regions such as in Ontario, Canada with the Edmonton Symptom Assessment System Revised for routine PROMs collection in hemodialysis routine care and should be encouraged as well in France [38]. In this regard, the French Society of Nephrology, Dialysis and Transplant (SFNDT) published in 2020 a new guideline recommending the use of EuroQol 5D and 12-Item Short Form Health Survey for outcome measures and e-Satis national public system for measuring patient satisfaction $[39,40]$.

Dedicated software linking patient registries in hemodialysis, collection of ePROMs for remote patient monitoring and measures of patient satisfaction may thus be used to ease and improve routine care as well as clinical and epidemiological research [41].

\section{Supplementary Information}

The online version contains supplementary material available at https://doi. org/10.1186/s12882-021-02551-3.

Additional file 1.

\section{Acknowledgements}

The authors thank the three centers and all the team M.Grange, F BelkahlaDelabruyere, F.Leveque (education nurse), AL.Camaroque (project manager) and S.Racanière (person in charge of relations with users) for their support for the management of the project and AcaciaTools for providing their medical writing and editing services.

\section{Authors' contributions}

$A G, J H$ analyzed and interpreted the results, MPR, AK, MLD, CD, PC, CL, ACB were major contributor in writing the manuscript. All authors read and approved the final manuscript.

Funding

No funding declared.

Availability of data and materials

The datasets used and/or analyzed during the current study are available from the corresponding author on reasonable request.

\section{Declarations}

Ethics approval and consent to participate

The study was declared to the data protection authority in France, known as the Commission Nationale de I'Informatique et des Libertés (CNIL), and was 
approved by the CPP Ile-de-France VII on 26 December 2019 in accordance with French regulations,

Informed consent was obtained form all participants involved in the study.

\section{Consent for publication}

Not applicable.

\section{Competing interests}

Authors declare there are no competing interests.

\section{Author details}

'Calydial Dialysis Department, Calydial, CH Vienne Lucien Hussel, Lucien Hussel Hospital, Vienne, France. ${ }^{2}$ AURAD-Aquitaine, Gradignan, France. ${ }^{3}$ Association pour l'Utilisation du Rein Artificiel en région Parisienne (AURA) Paris, Paris, France. ${ }^{4}$ Université Lyon, Université Claude Bernard Lyon 1, RESHAPE INSERM U1290, Lyon, France.

Received: 16 April 2021 Accepted: 22 September 2021 Published online: 30 October 2021

\section{References}

1. Theofilou P. Quality of life in patients undergoing hemodialysis or peritoneal dialysis treatment. J Clin Med Res. 2011;3(3):132-8.

2. Wyld M, Morton RL, Hayen A, Howard K, Webster AC. A systematic review and meta-analysis of utility-based quality of life in chronic kidney disease treatments. PLoS Med. 2012;9(9):e1001307. https://doi.org/10.1371/journ al.pmed.1001307 Epub 2012 Sep 11.

3. Weisbord SD, Fried LF, Mor MK, Resnick AL, Unruh ML, et al. Renal provider recognition of symptoms in patients on maintenance hemodialysis. Clin J Am Soc Nephrol. 2007;2(5):960-7.

4. Claxton RN, Blackhall L, Weisbord SD, Holley JL. Undertreatment of symptoms in patients on maintenance hemodialysis. J Pain Symptom Manag. 2010;39(2):211-8.

5. Chong K, Unruh M. Why does quality of life remain an under-investigated issue in chronic kidney disease and why is it rarely set as an outcome measure in trials in this population? Nephrol Dial Transplant. 2017;32(suppl_2):ii47-ii52.

6. Morton RL, Tong A, Howard K, Snelling P, Webster AC. The views of patients and carers in treatment decision making for chronic kidney disease: systematic review and thematic synthesis of qualitative studies. BMJ. 2010;340:c112.

7. Morton RL, Snelling P, Webster AC, Rose J, Masterson R, Johnson DW, et al. Factors influencing patient choice of dialysis versus conservative care to treat end-stage kidney disease. CMAJ. 2012;184(5):E277-83.

8. Dobrozsi S, Panepinto J. Patient-reported outcomes in clinical practice. Hematology Am Soc Hematol Educ Program. 2015;2015:501-6.

9. Anderson NE, Calvert M, Cockwell P, Dutton M, Aiyegbusi OL, Kyte D. Using patient-reported outcome measures (PROMs) to promote quality of care in the management of patients with established kidney disease requiring treatment with haemodialysis in the UK (PROM-HD): a qualitative study protocol. BMJ Open. 2018;8(10):e021532.

10. Anderson NE, Calvert M, Cockwell P, Dutton M, Kyte D. The use of patientreported outcomes in patients treated with maintenance hemodialysis: a perspective. Am J Kidney Dis. 2019 Sep;74(3):399-406.

11. Tong A, Sainsbury P, Craig J. Consolidated criteria for reporting qualitative research (COREQ): a 32-item checklist for interviews and focus groups. Int J Qual Health Care. 2007;19(6):349-57.

12. Lee KA, Hicks G, Nino-Murcia G. Validity and reliability of a scale to assess fatigue. Psychiatry Res. 1991;36(3):291-8.

13. Aaronson LS, Teel CS, Cassmeyer V, Neuberger GB, Pallikkathayil L, Pierce J, et al. Defining and measuring fatigue. Image J Nurs Sch. 1999;31(1):45-50

14. Cohen S, Kamarck T, Mermelstein R. A global measure of perceived stress. J Health Soc Behav. 1983;24(4):385-96 PMID: 6668417.

15. Cohen S, Williamson G, Spacapan S, Oskamp S. Perceived stress in a probability sample of the United States. The social Psychology of Health : Claremont symposium on Applied social psychology 1988.
16. Atkinson MJ, Lennox RD. Extending basic principles of measurement models to the design and validation of patient reported outcomes. Health Qual Life Outcomes. 2006;4:65.

17. Kemp J, Despres O, Dufour A. Unreliability of the visual analog scale in experimental pain assessment: a sensitivity and evoked potentials study. Pain Physician. 2012;15(5):E693-9.

18. Jhamb M, Weisbord SD, Steel JL, Unruh M. Fatigue in patients receiving maintenance dialysis: a review of definitions, measures, and contributing factors. Am J Kidney Dis. 2008;52(2):353-65. https://doi.org/10.1053/j.ajkd. 2008.05.005.

19. Murtagh FE, Addington-Hall J, Higginson IJ. The prevalence of symptoms in end-stage renal disease: a systematic review. Adv Chronic Kidney Dis. 2007;14(1):82-99.

20. Fleishman TT, Dreiher J, Shvartzman P. Patient-reported outcomes in maintenance hemodialysis: a cross-sectional, multicenter study. Qual Life Res. 2020;29(9):2345-54. https://doi.org/10.1007/s11136-020-02508-3.

21. Ju A, Teixeira-Pinto A, Tong A, Smith AC, Unruh M, et al. Validation of a Core patient-reported outcome measure for fatigue in patients receiving hemodialysis: the SONG-HD fatigue instrument. Clin J Am Soc Nephrol. 2020;15(11):1614-21.

22. Ramer SJ, Scherer JS. Moving the science of patient-reported outcome measures forward: measuring fatigue in hemodialysis patients. Clin J Am Soc Nephrol. 2020;15(11):1546-8.

23. Sondergaard H. Fatigue while undergoing long-term hemodialysis. Clin J Am Soc Nephrol. 2020;15(11):1539-40.

24. Rayner HC, Zepel L, Fuller DS, Morgenstern H, Karaboyas A, et al. Recovery time, quality of life, and mortality in hemodialysis patients: the Dialysis outcomes and practice patterns study (DOPPS). Am J Kidney Dis. 2014;64(1):86-94

25. Mirghaed MT, Sepehrian R, Rakhshan A, Gorji H. Sleep quality in Iranian hemodialysis patients: a systematic review and Meta-analysis. Iran J Nurs Midwifery Res. 2019;24(6):403-9.

26. Shen Q, Huang X, Luo Z, Xu X, Zhao X, He Q. Sleep quality, daytime sleepiness and health-related quality-of-life in maintenance haemodialysis patients. J Int Med Res. 2016:44(3):698-709.

27. Malhotra A, Loscalzo J. Sleep and cardiovascular disease: an overview. Prog Cardiovasc Dis. 2009;51(4):279-84.

28. Madsen MT, Huang C, Zangger G, Zwisler ADO, Gögenur I. Sleep disturbances in patients with coronary heart disease: a systematic review. J Clin Sleep Med. 2019;15(3):489-504.

29. Nimmo A, Bell S, Brunton C, Campbell J, Doyle A, MacKinnon B, Peel RK, Robertson S, Shilliday I, Spalding E, Traynor JP, Metcalfe W; Scottish renal registry. Collection and determinants of patient reported outcome measures in haemodialysis patients in Scotland. QJM. 2018;111(1):15-21.

30. Lassalle M, Monnet E, Ayav C, Hogan J, Moranne O. Couchoud C; REIN registry. 2017 annual report digest of the renal epidemiology information network (REIN) registry. Transpl Int. 2019;32(9):892-902.

31. Couchoud C, Gharbi MB. Advocacy for renal replacement therapy: the role of renal registries. Clin Kidney J. 2020;13(5):742-4.

32. Agence de la biomédecine. Registre français des traitements de suppléance de l'insuffisance rénale chronique Rapport 2018. 2018. Accessible at: https://www.agence-biomedecine.fr/IMG/pdf/rapport_rein_2018_v2. pdf. Last Accessed on 1 Dec 2020.

33. van der Willik E, Hemmelder M, Bart H, van Ittersum F, Hoogendijkvan den Akker J, et al. Routinely measuring symptom burden and health-related quality of life in dialysis patients: first results from the Dutch registry of patient-reported outcome measures,. Clin Kidney J. 2021;14(6):1535-1544,

34. Sloan JA, Liu H, Satele DV, Puttabasavaiah S, Kaur JS, Hubbard J, et al. Prognostic significance of baseline fatigue for overall survival: a patientlevel meta-analysis of 43 oncology clinical trials with 3915 patients. Trends Cancer Res. 2017;12:97-110.

35. Jacobson J, Ju A, Baumgart A, Unruh M, O'Donoghue D, et al. Patient perspectives on the meaning and impact of fatigue in hemodialysis: a systematic review and thematic analysis of qualitative studies. Am J Kidney Dis. 2019;74(2):179-92.

36. Nixon AC, Bampouras TM, Pendleton N, Mitra S, Brady ME, Dhaygude AP Frailty is independently associated with worse health-related quality of life in chronic kidney disease: a secondary analysis of the frailty assessment in chronic kidney disease study. Clin Kidney J. 2019;13(1):85-94. 
37. Picariello F, Norton S, Moss-Morris R, Macdougall IC, Chilcot J. Fatigue in prevalent Haemodialysis patients predicts all-cause mortality and kidney transplantation. Ann Behav Med. 2019;53(6):501-14.

38. Evans JM, Glazer A, Lum R, Heale E, MacKinnon M, Blake PG, et al. Implementing a patient-reported outcome measure for hemodialysis patients in routine clinical care: perspectives of patients and providers on ESASr:renal. Clin J Am Soc Nephrol. 2020;15(9):1299-309.

39. Ayav C, Couchoud C, Sautenet B, Lobbedez T, Sens F, Moranne O; Commission épidémiologie et santé publique de la Société francophone de néphrologie, dialyse et transplantation. Le recueil en routine de données de santé perçue à l'ère du paiement à la qualité : préconisations de la Commission épidémiologie et santé publique de la SFNDT [Routine collection of perceived health data in the era of payment for quality: Recommendations by the Epidemiology and public health commission of the SFNDT]. Nephrol Ther. 2020:S1769-7255(20)30102-4

40. Gloanec M, Capuano F, Sainte-Croix D, May-Michelangeli L. L'enquête nationale e-Satis, mesurer la satisfaction et l'expérience des patients. Soins. 2018;63(829):23-6.

41. Whitlow M, Wallace E. Remote Patient Monitoring: An Important Tool in Advancing Home Dialysis. Kidney Med. 2019;1(6):327-.

\section{Publisher's Note}

Springer Nature remains neutral with regard to jurisdictional claims in published maps and institutional affiliations.
Ready to submit your research? Choose BMC and benefit from:

- fast, convenient online submission

- thorough peer review by experienced researchers in your field

- rapid publication on acceptance

- support for research data, including large and complex data types

- gold Open Access which fosters wider collaboration and increased citations

- maximum visibility for your research: over $100 \mathrm{M}$ website views per year

At BMC, research is always in progress.

Learn more biomedcentral.com/submissions 